PROCEEDINGS OF THE

AMERICAN MATHEMATICAL SOCIETY

Volume 128, Number 3, Pages 909-918

S 0002-9939(99)05038-8

Article electronically published on May 6, 1999

\title{
AN OPEN SET OF MAPS FOR WHICH EVERY POINT IS ABSOLUTELY NONSHADOWABLE
}

\author{
GUO-CHENG YUAN AND JAMES A. YORKE
}

(Communicated by Mary Rees)

\begin{abstract}
We consider a class of nonhyperbolic systems, for which there are two fixed points in an attractor having a dense trajectory; the unstable manifold of one has dimension one and the other's is two dimensional. Under the condition that there exists a direction which is more expanding than other directions, we show that such attractors are nonshadowable. Using this theorem, we prove that there is an open set of diffeomorphisms (in the $C^{r}$ topology, $r>1$ ) for which every point is absolutely nonshadowable, i.e., there exists $\epsilon>0$ such that, for every $\delta>0$, almost every $\delta$-pseudo trajectory starting from this point is $\epsilon$-nonshadowable.
\end{abstract}

\section{INTRODUCTION}

In study of chaotic systems, a researcher is often obliged to rely on numerical simulations because direct analytical methods are not available. To ensure their validity, it is crucial that numerical trajectories stay close to, in other words, they are shadowed (see Section 2 for definition) by, true trajectories; otherwise, the meaning of numerical results is far from obvious. Although compact hyperbolic invariant sets are shadowable as proved by Anosov [2] and Bowen [4], virtually all chaotic attractors that scientists encounter are nonhyperbolic. For example, the "Hénon" strange attractors constructed by Benedicks and Carleson [3] are not hyperbolic: the angle between stable and unstable manifolds is not bounded away from zero. There seems to be a feeling among many mathematicians that nonhyperbolicity generally implies nonshadowability, but there are counterexamples. Nonhyperbolic attractors can have the shadowing property. Let $F_{\lambda}$ be a one parameter family of maps or diffeomorphisms with a periodic attractor that undergoes a cascade of period doublings, limiting on the so-called "Feigenbaum" parameter $\lambda_{c}$ (which was first described by Myrberg [17]). The corresponding "Feigenbaum" attractor at this value is not hyperbolic, but it does satisfy the shadowing property. Furthermore, if the above example lies on a space $M_{1}$, and on another space $M_{2}$ there is a hyperbolic attractor, then the attractor for the product system is chaotic and has the shadowing property, but the attractor is not hyperbolic. For a simpler example, Coven, Kan and Yorke [9] have shown that tent maps are shadowable for all parameter values (i.e., the absolute value of the slopes) between 1 and 2 except for a set having zero Lebesgue measure. (Indeed, this paper discusses tent maps with

Received by the editors November 6, 1997 and, in revised form, April 21, 1998.

1991 Mathematics Subject Classification. Primary 58F13; Secondary 58F12, 58F14, 58F15.

This research was supported by the National Science Foundation and Department of Energy. 
slopes between $\sqrt{2}$ and 2 , though it is trivially extendible to slopes between 1 and 2. They also show that the set of parameters for which the map is nonshadowable is uncountable.) In their case, the attractor is the whole space. Similarly, in the set of parameter values where the logistic map has a chaotic attractor there is a dense, uncountable set for which it is shadowable and a dense uncountable set for which it is not. In the window of parameter values with an attracting periodic orbit, it is shadowable; see Smale and Williams [23] for the period 3 case, where they show the invariant set of points not in the period 3 basin of the attractor is hyperbolic.

Several papers $[12,13,21,7,8,5,6,20]$ have given methods to rigorously verify whether numerical trajectories are shadowed by true trajectories. Results obtained from applying such methods to nonhyperbolic maps on one and two dimensional phase spaces $[12,13,21]$ suggest that such systems are nonshadowable. The critical point of the logistic map is analogous to points of the chaotic attractors of Hénon maps where the stable and unstable sets are tangent. However, the Hénon attractor appears to have uncountably many such points, so the analysis is more complicated and the shadowing properties are yet unresolved.

Our goal is to discuss another mechanism for nonshadowability which we call "dimension variability", i.e., an attractor (with a dense trajectory) has at least two hyperbolic periodic orbits whose unstable manifolds have different dimensions. Using this phenomenon, we present an open set of maps (or diffeomorphisms) for which every point is absolutely nonshadowable (see Section 2 for definition). In the next section we will analyze geometrically how dimension variability is an obstacle for shadowing. Some of our ideas have been sketched in Poon et al. [18].

Dimension variability is related to studies of finite shadowing time - i.e., starting from an initial point, for how long can the pseudo trajectory be shadowed by a true trajectory (the shadowing distance is comparable to the size of the attractor) - for nonhyperbolic systems. It has been conjectured $[12,13]$ that for typical dissipative maps being iterated with numerical accuracy $\delta$, the average shadowing time is of the order $1 / \sqrt{\delta}$. This conjecture is supported by numerical studies of the logistic map and Hénon map. Recent studies discuss a new type of map in which almost every trajectory has the following property. The Lyapunov exponents are non-zero, but there are arbitrarily long segments of the trajectory for which the number of the approximate "finite-time" Lyapunov exponents that are positive is different from the number of actual positive exponents. For such maps, the average shadowing time can be much shorter, meaning that the shadowing difficulty is more serious than it is for the logistic map and Hénon map. Systems with dimension variability have this property, because almost every trajectory has arbitrarily long segments in which it remains near each of the periodic orbits.

\section{MAIN THEOREM}

Let $M$ be an $m$-dimensional Riemannian manifold and $f: M \rightarrow M$ be a $C^{1}$ map. Let $A$ be a compact invariant set; we say $A$ is Lyapunov stable if there exists a family of neighborhoods $U_{i}$ of $A$ such that $\bigcap_{i} \overline{U_{i}}=A$ and $f\left(U_{i}\right) \subset U_{i}$ for all $i$. We say $A$ is an attractor, if i) there exists an neighborhood $U$ such that, for every $x \in U$, the positive limit set $\omega(x) \subset A$; ii) $A$ is Lyapunov stable; and iii) there exists $p \in M$ such that $\omega(p)=A$. If $A$ is an attractor, we say the set $\{p \in M: \omega(p) \subset A\}$ is the basin of $A$. Notice that the usual definition of an attractor only requires the first condition, but then it would include the pathological cases where nearby 
orbits have many intermediate iterates that stay away from the invariant set. We exclude such pathological cases by adding condition ii) in our definition. We also add condition iii) to avoid unnecessarily large attractors. For example, the whole manifold is an attractor under the usual definition, whereas under our definition it is an attractor only if there exists an orbit that is dense everywhere in the manifold.

Given $\delta, \epsilon>0$, we say $\bar{x}=\left(x_{i}\right)_{i=a}^{b}$ is a $\delta$-pseudo trajectory if $d\left(f\left(x_{i}\right), x_{i+1}\right)<\delta$ whenever $a \leq i<b$. Let $\Omega_{x, \delta}$ be the set of $\delta$-pseudo trajectories that start from $x$. If we consider $x_{i+1}$ as being chosen at random from a uniform distribution in $B(x, \delta)$ (from now on, we use $B(x, \delta)$, resp. $B(V, \delta)$, to denote the $\delta$-neighborhood of a point $x$, resp. a subset $V$ of $M$ ), then $\Omega_{x, \delta}$ forms a Markov chain with each $\delta$-pseudo trajectory in $\Omega_{x, \delta}$ as a sample sequence [10]. We say "almost every $\delta$ pseudo trajectory" has a property $\mathcal{P}$ if the event $\mathcal{P}$ occurs with probability 1 for this Markov chain. Similar ideas have been discussed by Young [24] and Kifer [15].

Given $\gamma>0$, we say a pseudo orbit $\left(x_{i}\right)_{i=0}^{\infty}$ comes within $\gamma$ of a finite pseudo orbit $\left(y_{i}\right)_{i=0}^{n}$ if there exists $\ell \geq 0$ such that $d\left(x_{\ell+i}, y_{i}\right)<\gamma$, for $1 \leq i \leq n$. We have the following observation.

Proposition 2.1. Let $A$ be an attractor and $U$ be the basin of $A$. Let $x \in U$. Assume that $\delta>0$ is sufficiently small that there exists a compact neighborhood $V \subset U$ of $A$ such that $x \in V$ and $f(B(V, \delta)) \subset V$. Let $\bar{y}:=\left(y_{0}, \ldots, y_{n}\right)$ be a $\delta$ pseudo trajectory in $B(A, \delta)$. Then for each $\gamma>0$, almost every $\bar{x}:=\left(x_{i}\right)_{i=0}^{\infty} \in \Omega_{x, \delta}$ comes within $\gamma$ of $\bar{y}$.

Proof. Denote by $\mathcal{E}_{x}$ the event that a pseudo orbit $\bar{x} \in \Omega_{x, \delta}$ comes within $\gamma$ of $\bar{y}$. For each $z \in V$, there exist an open neighborhood $U_{z}$ of $z$, a positive number $\sigma_{z}$, and a positive integer $n_{z}$ such that, for each point $z^{\prime} \in U_{z}$, the probability for a finite $\delta$-pseudo trajectory $\left(z_{i}\right)_{i=0}^{n_{z}}$ with $z_{0}=z^{\prime}$ to come within $\gamma$ of $\bar{y}$ is greater than $\sigma_{z}$. Since $V$ is compact, there exists a finite set $\left\{z^{(1)}, \ldots, z^{(k)}\right\} \subset V$ such that $\bigcup_{i=1}^{k} U_{z^{(i)}} \supset V$. This implies that there exist $\sigma^{*}>0$ and $n^{*}>0$ such that, for every $z \in V$, the probability for a $\delta$-pseudo trajectory $\left(z_{i}\right)_{i=0}^{n^{*}}$ with $z_{0}=z$ to come within $\gamma$ of $\bar{y}$ is greater than $\sigma^{*}$. Note that if $x \in V$ and $\bar{x} \in \Omega_{x, \delta}$, then $\left\{x_{i}\right\}_{i=0}^{\infty} \subset V$. Each of the finite $\delta$-pseudo trajectories $\left(x_{i}\right)_{i=j n^{*}}^{j n^{*}+n^{*}-1}, 0 \leq j<\infty$, comes within $\gamma$ of $\bar{y}$ with probability greater than $\sigma^{*}$. Since there are infinitely many independent trials, $P\left(\mathcal{E}_{x}\right)=1$.

A $\delta$-pseudo trajectory $\bar{x}:=\left(x_{i}\right)_{i=a}^{b}$ is $\epsilon$-shadowed by $y$ if $d\left(x_{i}, f^{i}(y)\right)<\epsilon$ when $a \leq i \leq b$. We say a point $x$ is absolutely nonshadowable if there exists $\epsilon>0$ such that, for every $\delta>0$, almost every $\delta$-pseudo trajectory $\left(x_{i}\right)_{i=0}^{\infty} \in \Omega_{x, \delta}$ is $\epsilon$ nonshadowable, i.e., it cannot be $\epsilon$-shadowed by any true trajectory. Of course, $\Omega_{x, \delta}$ includes true trajectories, and those are trivially shadowable; hence we can only require that almost every $\delta$-pseudo trajectory is $\epsilon$-nonshadowable.

Before we state our theorem, we need to introduce some notions, which play an important role in the proof of the well-known Hadamard-Perron Theorem [11]. (An excellent presentation of the proof can be found in [14], pages 242-257.) Let $S_{k}=\left\{(u, v) \in \mathbf{R}^{k} \oplus \mathbf{R}^{m-k}:|u| \leq|v|\right\}$. We say $S_{k}$ is the standard k-cone in $\mathbf{R}^{m}$. A set $C_{0} \subset \mathbf{R}^{m}$ is said to be a k-cone if $C_{0}$ is isomorphic to $S_{k}$ up to a linear change of variables. Let $\mathcal{C} \subset T M$. We write $C_{x}:=\mathcal{C} \cap T_{x} M$. Assume for $x \in A, C_{x}$ is nonempty and $x \longmapsto C_{x}$ is continuous on $A$ in the Hausdorff metric. We say $\mathcal{C}$ is a k-cone field on $A$ if, for each $x \in A, C_{x}$ is a k-cone. We say $\mathcal{C}$ is positively invariant if $(D f) C_{x} \subset \operatorname{int} C_{f(x)} \cup\{0\}$ for all $x \in A$. 
We say an invariant set $A$ has dimension variability if there exist hyperbolic periodic points $p_{1}, p_{2}$ in $A$, such that $0<\operatorname{dim}\left(W^{u}\left(p_{1}\right)\right)<\operatorname{dim}\left(W^{u}\left(p_{2}\right)\right)$. Below we assume for convenience that these orbits are fixed points.

Theorem 2.2. Let $f: M \rightarrow M$ be a $C^{1}$ map. Assume $f$ satisfies the following properties:

1. $f$ has an attractor $A$, and $U$ is its basin;

2. (dimension variability) there exist hyperbolic fixed points $p_{1}, p_{2}$ in $A$, such that $0<\operatorname{dim}\left(W^{u}\left(p_{1}\right)\right)<\operatorname{dim}\left(W^{u}\left(p_{2}\right)\right)$;

3. $\overline{W^{u}\left(p_{1}\right)}=A$;

4. write $k:=\operatorname{dim}\left(W^{u}\left(p_{1}\right)\right)$; there exists a positively invariant $k$-cone field $\mathcal{C}$ on A.

Then every point in $U$ is absolutely nonshadowable.

Remark 2.3. If $f$ is a diffeomorphism, then the following condition is equivalent to Condition 4 in Theorem 2.2:

$4^{\prime}$. There exists a continuous splitting $T_{A} M=E_{1} \oplus E_{2}$, where $\operatorname{dim}\left(E_{1}\right)=$ $\operatorname{dim}\left(W^{u}\left(p_{1}\right)\right)$, and positive constants $K, \lambda$ and $\mu$ with $\lambda<\mu$, such that: i) $D f\left(E_{i}\right)=$ $E_{i}, i=1,2$; ii) for all $v \in E_{1}$ and $n \geq 0,\left|D f^{-n}(v)\right| \leq K \mu^{-n}|v|$; and iii) for all $v \in E_{2}$ and $n \geq 0,\left|D f^{n}(v)\right| \leq K \lambda^{n}|v|$. Note in particular $\lambda$ need not be less than 1.

Remark 2.4. We have cited literature discussing shadowing failure due to tangencies of stable and unstable manifolds. Condition 4 guarantees there are no tangencies. Hence dimension variability is a distinct mechanism. In particular, Condition 4 prevents $W^{u}\left(p_{1}\right)$ from being tangent to $W^{s}\left(p_{2}\right)$ at any point in $W^{u}\left(p_{1}\right) \cap W^{s}\left(p_{2}\right)$. If $f$ is a diffeomorphism, then, at each $x \in W^{u}\left(p_{1}\right) \cap W^{s}\left(p_{2}\right), W^{u}\left(p_{1}\right)$ is tangent to $E_{1}(x)$, and $W^{s}\left(p_{2}\right)$ is tangent to a subspace of $E_{2}(x)$. Since the angle between $E_{1}$ and $E_{2}$ is bounded away from $0, W^{u}\left(p_{1}\right)$ cannot be tangent to $W^{s}\left(p_{2}\right)$ at any point.

Remark 2.5. Condition 4 is also a uniform condition to make it possible for us to obtain a proof. We do not know if the result is true without Condition 4.

To simplify notation, we prove Theorem 2.2 for the case $\operatorname{dim}\left(W^{u}\left(p_{1}\right)\right)=1$ and $\operatorname{dim}\left(W^{u}\left(p_{2}\right)\right)=2$. The proof for the other cases follows in the same manner.

For the rest of this section, we assume all the conditions in Theorem 2.2 are satisfied. We need some notation before proving the theorem. For $i=1,2$, write $E^{u}\left(p_{i}\right) \subset T_{p_{i}} M$ for the unstable space (i.e., with respect to $D f$ ) at $p_{i}$, and $W^{u}\left(p_{i}\right)$ for the unstable manifold of $p_{i}$. For $\epsilon>0$, let $W_{\epsilon}^{u}\left(p_{1}\right)$ be the local unstable manifold of $p_{1}$. For $x \in A$, write $C(x, \epsilon):=\left\{u \in C_{x}:|u| \leq \epsilon\right\}$, and let $\exp _{x}: T_{x} M \rightarrow M$ be the exponential map induced by the Riemannian metric. If $\epsilon$ is sufficiently small, then $\exp _{x}$ maps $C(x, \epsilon)$ diffeomorphically to a subset $\exp _{x} C(x, \epsilon)$ in $M$. For convenience, we do not distinguish between $\exp _{x} C(x, \epsilon)$ and $C(x, \epsilon)$ in our notation.

The following lemmas are needed in the proof of Theorem 2.2.

Lemma 2.6. (a) There exists $\epsilon_{0}>0$ such that if $x \in A$, then

$$
f(C(x, \epsilon)) \cap B(f(x), \epsilon) \subset \operatorname{int}(C(f(x), \epsilon)) \cup\{f(x)\},
$$

for each $0<\epsilon<\epsilon_{0}$.

(b) Moreover, there exists $\alpha>0$, such that if $x \in A, w \in B(f(x), \epsilon)$, and $w \notin \operatorname{int} C(f(x), \epsilon)$, then $d(w, f(C(x, \epsilon))) \geq \alpha d(w, f(x))$. 
Proof. Given $x \in A, \epsilon>0$, and $v \in T_{x} M,|v|=1$, let $l(x, v, \epsilon)=\left\{\exp _{x}(t v): 0 \leq\right.$ $t \leq \epsilon\}$. If $v \in C_{x}$, then $D f_{x} v \in \operatorname{int} C_{f(x)}$. Therefore there exists $\epsilon_{x, v}>0$ such that $f(l(x, v, \epsilon)) \cap B(f(x), \epsilon) \subset \operatorname{int} C(f(x), \epsilon) \cup\{f(x)\}$ for $0<\epsilon<\epsilon_{x, v}$. By continuity, there exists $\epsilon_{x}>0$ such that, for all $v \in C_{x}$ with $|v|=1, f(l(x, v, \epsilon)) \cap B(f(x), \epsilon) \subset$ int $C(f(x), \epsilon) \cup\{f(x)\}$ for $0<\epsilon<\epsilon_{x}$. Also by continuity, there exists $\epsilon_{0}$ such that, for all $x \in A$ and $0<\epsilon<\epsilon_{0}, f(C(x, \epsilon)) \cap B(f(x), \epsilon) \subset$ int $C(f(x), \epsilon) \cup\{f(x)\}$.

In the following we prove the second part of the lemma. Since $D f_{x} C_{x} \subset$ int $_{f(x)} \cup\left\{0_{f(x)}\right\}$ and $D f_{x}$ is a linear map, there exists $\gamma$ such that if $v \in T_{f(x)} M$ and $v \notin i n t C_{f(x)}$, then $d\left(v, D f_{x} C_{x}\right) \geq \gamma|v|$. Since $\exp _{x}$ is a local diffeomorphism, the previous argument implies statement $(b)$.

Lemma 2.7. There exist $\epsilon_{0}, \eta>0$ and $\lambda>1$ with the following properties.

(a). On $B\left(p_{2}, \epsilon_{0}\right)$ there exists an invariant two dimensional $C^{0}$-(unstable) foliation such that if $y, z$ are in the same leaf and $\left\{f^{i}(y), f^{i}(z)\right\}_{i=0}^{n} \subset B\left(p_{2}, \epsilon_{0}\right)$, then

for $0 \leq i \leq n$.

$$
d\left(f^{i}(y), f^{i}(z)\right) \geq \eta \lambda^{i} d(y, z)
$$

(b). On $B\left(p_{1}, \epsilon_{0}\right)$ there exists an invariant $(m-1)$-dimensional $C^{0}$-(stable) foliation such that if $y, z$ are in the same leaf and $\left\{f^{i}(y), f^{i}(z)\right\}_{i=0}^{n} \subset B\left(p_{1}, \epsilon_{0}\right)$, then

$$
d\left(f^{i}(y), f^{i}(z)\right) \leq\left(\eta \lambda^{i}\right)^{-1} d(y, z)
$$

for $0 \leq i \leq n$.

Proof. We only prove the first part. Similar arguments apply to the second part.

If $f$ is a linear map, then we define the leaf through each $x \in B\left(p_{2}, \epsilon_{0}\right)$ by $x+W^{u}\left(p_{2}\right)$. The foliation thus defined satisfies property (a).

In general, $f$ is locally conjugate to a linear map. Note that property (a) is preserved by conjugacies. The proof is complete.

The foliations given by Lemma 2.7 are not unique. From now on, we fix one unstable foliation on $B\left(p_{2}, \epsilon_{0}\right)$ and one stable foliation on $B\left(p_{1}, \epsilon_{0}\right)$. We write $W^{u}\left(y ; p_{2}, \epsilon_{0}\right)$ for the leaf of this unstable foliation through $y \in B\left(p_{2}, \epsilon_{0}\right)$ and $W^{s}\left(z ; p_{1}, \epsilon_{0}\right)$ for the leaf of this stable foliation through $z \in B\left(p_{1}, \epsilon_{0}\right)$.

Lemma 2.8. There exists $\epsilon_{0}>0$ such that the following statement is true for each $0<\epsilon<\epsilon_{0}$ and $\delta>0$.

Let $n \in \boldsymbol{N}$ and $x \in B\left(p_{2}, \epsilon\right)$ be such that $x, f(x), \ldots, f^{n}(x) \in B\left(p_{2}, \epsilon\right)$. Then there exists $w \in W^{u}\left(x ; p_{2}, \epsilon\right)$ such that i) $d(x, w) \leq \delta$; and ii) $f^{i}(w) \notin C\left(f^{i}(x), \epsilon\right)$, for $0 \leq i \leq n$. Furthermore, if $\delta$ is sufficiently small, then $w$ can be chosen so that $d(x, w) \geq \delta / 2$.

Proof. There exists $\epsilon_{0}>0$ such that, for each $x \in M, f$ maps $B\left(x, \epsilon_{0}\right)$ diffeomorphically onto its image $f\left(B\left(x, \epsilon_{0}\right)\right)$. Let $x, f(x), \ldots, f^{n}(x) \in A \cap B\left(p_{2}, \epsilon_{0}\right)$. Write $W:=$ $\bigcap_{i=0}^{n} f^{-i}\left(B\left(f^{i}(x), \epsilon_{0}\right)\right)$. Then $W$ contains a neighborhood of $x$. Therefore $f^{n}(W)$ contains a neighborhood of $f^{n}(x)$. In particular, there exists a curve $l \subset W$ passing through $f^{n}(x)$ such that $l \subset W^{u}\left(f^{n}(x) ; p_{2}, \epsilon\right)$ and $l \cap C\left(f^{n}(x), \epsilon_{0}\right)=\left\{f^{n}(x)\right\}$. Let $l^{\prime}=f^{-n}(l) \cap W$. Then $l^{\prime}$ is a curve passing through $x$ and $l^{\prime} \subset W^{u}\left(x ; p_{2}, \epsilon\right)$. For each $w \in l^{\prime}$ different from $x, f^{i}(w)(0 \leq i \leq n)$ is not contained in $C\left(f^{i}(x), \epsilon\right)$. In particular, $w$ can be chosen so that $d(w, x) \leq \delta$. If $\delta$ is sufficiently small, then $\delta$ can be chosen so that $d(w, x) \geq \delta / 2$. 
We say a $\delta$-pseudo trajectory $\bar{x}$ is elementary if there exists $i_{0}$, such that $f\left(x_{i}\right)=x_{i+1}$ for all $i$ except for $i=i_{0}$. The next lemma is the key step toward the final proof of Theorem 2.2.

Lemma 2.9. There exist $\epsilon_{0}>0$ and $n_{0}>0$ such that for all $0<\epsilon^{\prime}<\epsilon_{0}$ and $\delta>0$, there exist $x \in W_{\epsilon^{\prime}}^{u}\left(p_{1}\right)$ and an elementary $\delta$-pseudo trajectory $\bar{x}=\left(x_{i}\right)_{i=0}^{n_{0}}$, where $x_{0}=x$, such that $\bar{x}$ cannot be $\epsilon^{\prime}$-shadowed by any $y \in C\left(x, \epsilon^{\prime}\right)$.

Proof. Let $\epsilon_{0}$ be a small number such that the next two conditions are satisfied:

1. Lemmas $2.6-2.8$ hold.

2. $f$ restricted to $B\left(p_{1}, \epsilon_{0}\right)$ is one-to-one, and $W_{\epsilon_{0}}^{u}\left(p_{1}\right) \subset f\left(W_{\epsilon_{0}}^{u}\left(p_{1}\right)\right)$.

Fix $\epsilon^{\prime} \in\left(0, \epsilon_{0}\right)$. Given $\delta>0$, there exists $n_{1}$, such that $\eta \alpha \delta \lambda^{n_{1}}>2 \epsilon^{\prime}$, where $\alpha$ is as in Lemma 2.6 and $\eta$ and $\lambda$ are as in Lemma 2.7. Let $s>0$ be sufficiently small, such that

$$
\bigcup_{i=0}^{n_{1}} f^{i}\left(B\left(p_{2}, s\right)\right) \subset B\left(p_{2}, \epsilon^{\prime}\right)
$$

Choose $x^{\prime} \in W^{u}\left(p_{1}\right)$, such that $f\left(x^{\prime}\right) \in B\left(p_{2}, s\right)$.

In the following we will introduce a small perturbation at $f\left(x^{\prime}\right)$ such that the perturbed trajectory is not shadowable. Note that $W^{u}\left(p_{2}\right)$ is two dimensional whereas $W^{u}\left(p_{1}\right)$ is only one dimensional. When the trajectory is perturbed away from $W^{u}\left(p_{1}\right)$, the trajectory diverges from $W^{u}\left(p_{1}\right)$. We will see this leads to nonshadowability.

We first prove that there exists a $\delta$-pseudo trajectory starting from $x^{\prime}$ which can not be $\epsilon^{\prime}$-shadowed by any $y \in C\left(x^{\prime}, \epsilon^{\prime}\right)$. By Lemma 2.8 , for every $\delta>0$, there exists $w \in W^{u}\left(f\left(x^{\prime}\right) ; p_{2}, \epsilon^{\prime}\right)$ such that i) $d\left(f\left(x^{\prime}\right), w\right) \leq \delta$; and ii) $f^{i}(w) \notin C\left(f^{i+1}\left(x^{\prime}\right), \epsilon^{\prime}\right)$ for $0 \leq i \leq n_{1}$. For the purpose of shadowing, we let $d\left(f\left(x^{\prime}\right), w\right) \geq \delta / 2$. Therefore

$$
\begin{aligned}
d\left(f^{n_{1}}(w), f^{n_{1}+1}\left(C\left(x^{\prime}, \epsilon^{\prime}\right)\right)\right) & \geq d\left(f^{n_{1}}(w), f\left(C\left(f^{n_{1}}\left(x^{\prime}\right), \epsilon^{\prime}\right)\right)\right) \\
& \geq \alpha d\left(f^{n_{1}} w, f^{n_{1}+1}\left(x^{\prime}\right)\right) \\
& \geq \alpha \eta \lambda^{n_{1}} d\left(w, f\left(x^{\prime}\right)\right) \\
& \geq \frac{\alpha \eta \lambda^{n_{1}} \delta}{2} \\
& >\epsilon^{\prime} .
\end{aligned}
$$

In the above estimation, we have used Lemmas 2.6-2.8.

The elementary $\delta$-pseudo trajectory $\left(x^{\prime}, w, f(w), \cdots, f^{n_{1}}(w)\right)$ cannot be $\epsilon^{\prime}$ shadowed by any $y \in C\left(x^{\prime}, \epsilon^{\prime}\right)$.

If $x^{\prime} \in W_{\epsilon^{\prime}}^{u}\left(p_{1}\right)$, then by letting $x$ equal $x^{\prime}$ we are done. Otherwise, there exist $x \in W_{\epsilon^{\prime}}^{u}\left(p_{1}\right)$ and $n_{2}>0$, such that $x^{\prime}=f^{n_{2}}(x)$. Define $x_{i}=f^{i}(x)$ for $0 \leq i \leq n_{2}$, and $x_{i}=f^{i-n_{2}-1}(w)$ for $i \geq n_{2}+1$. Let $n_{0}=n_{1}+n_{2}+1$; then $\bar{x}=\left(x_{i}\right)_{i=0}^{n_{0}}$ cannot be $\epsilon^{\prime}$-shadowed by any $y \in C\left(x, \epsilon^{\prime}\right)$.

Let $\epsilon_{0}, \epsilon^{\prime}, x, \bar{x}$ and $n_{0}$ be as in Lemma 2.9. For $i<0$, let $x_{i}$ be the unique point in $W_{\epsilon_{0}}^{u}\left(p_{1}\right) \cap f^{-1}\left(x_{i+1}\right)$. Define $\bar{x}_{i}:=\left(x_{j}\right)_{j=i}^{n_{0}}$. Since $x \in W^{u}\left(p_{1}\right), x_{i} \rightarrow p_{1}$ as $i \rightarrow-\infty$. Note that, for each $i<0, \bar{x}_{i}$ cannot be $\epsilon^{\prime}$-shadowed by any $y \in C\left(x_{i}, \epsilon^{\prime}\right)$.

The next two lemmas extend the result in Lemma 2.9. They are needed to finish the proof.

Lemma 2.10. Let $\epsilon_{0}, x, \bar{x}$ and $n_{0}$ be as in Lemma 2.9. Then there exists $\sigma>0$, such that $\bar{x}$ is not $\epsilon$-shadowed by any $y \in B(C(x, \epsilon), \sigma)$, for $0<\epsilon<\frac{\epsilon_{0}}{2}$. 
Proof. Fix $\epsilon^{\prime} \in\left(0, \epsilon_{0}\right)$. Let $\sigma>0$ be sufficiently small such that

$$
\max _{1 \leq i \leq n_{0}} d\left(f^{i}(y), f^{i}(z)\right)<\epsilon^{\prime} / 2
$$

whenever $d(y, z)<\sigma$. If $y \in B\left(C\left(x, \epsilon^{\prime}\right), \sigma\right)$, then there exists $z \in C\left(x, \epsilon^{\prime}\right)$ such that $d(y, z)<\sigma$. From Lemma $2.9, d\left(f^{n_{0}}(z), x_{n_{0}}\right)>\epsilon^{\prime}$. Therefore

$$
\begin{aligned}
d\left(f^{n_{0}}(y), x_{n_{0}}\right) & \geq d\left(f^{n_{0}}(z), x_{n_{0}}\right)-d\left(f^{n_{0}}(y), f^{n_{0}}(z)\right) \\
& >\epsilon^{\prime}-\frac{\epsilon^{\prime}}{2}=\frac{\epsilon^{\prime}}{2} .
\end{aligned}
$$

$\bar{x}$ is not $\epsilon^{\prime} / 2$-shadowed by $y$. Let $\epsilon=\epsilon^{\prime} / 2$; then the proof is complete.

Lemma 2.11. Define $\bar{x}_{i}, i<0$, as in the paragraph that follows Lemma 2.9. Let $\epsilon_{0}$ and $\epsilon$ be as in Lemma 2.10. Then there exists $n_{3}>0$, such that $\bar{x}_{-n_{3}}$ cannot be $\epsilon$-shadowed by any $y \in M$.

Proof. Given $y \in B\left(p_{1}, \epsilon\right)$ and $n>0$ such that $\left\{f^{i}(y)\right\}_{i=0}^{n} \subset B\left(p_{1}, \epsilon_{0}\right)$, there exists $z \in W^{s}\left(y ; p_{1}, \epsilon\right) \cap W_{\epsilon}^{u}\left(p_{1}\right)$ such that $\left\{f^{i}(z)\right\}_{i=0}^{n} \subset B\left(p_{1}, \epsilon_{0}\right)$. By Lemma 2.7, $d\left(f^{i}(y), f^{i}(z)\right) \leq\left(\eta \lambda^{i}\right)^{-1} d(y, z)$ for $0 \leq i \leq n$. Therefore $d\left(f^{i}(y), W_{\epsilon}^{u}\left(p_{1}\right)\right) \leq$ $\left(\eta \lambda^{i}\right)^{-1} \epsilon$.

Let $y \in M$ and $n_{3} \geq 1$ be such that $d\left(f^{i}(y), x_{i-n_{3}}\right)<\epsilon$ for $0 \leq i \leq n_{3}$. Note that $x \in W_{\epsilon}^{u}\left(p_{1}\right)$. From previous arguments,

$$
d\left(f^{n_{3}}(y), C(x, \epsilon)\right) \leq d\left(f^{n_{3}}(y), W_{\epsilon}^{u}\left(p_{1}\right)\right) \leq\left(\eta \lambda^{n_{3}}\right)^{-1} \cdot \epsilon .
$$

We can choose $n_{3}$ to be sufficiently large that $\left(\eta \lambda^{n_{3}}\right)^{-1} \cdot \epsilon<\sigma$. Apply Lemma 2.10; then $\bar{x}$ is not $\epsilon$-shadowed by $f^{n_{3}}(y)$. Therefore $\bar{x}_{-n_{3}}$ is not $\epsilon$-shadowed by $y$.

Proof of Theorem 2.2. In the previous lemmas, we have proved that the $\delta$-pseudo trajectory

$$
\bar{x}_{-n_{3}}=\left(x_{-n_{3}}, x_{-n_{3}+1}, \ldots, x, w, f(w), \ldots, f^{n_{1}}(w)\right)
$$

cannot be $\epsilon$-shadowed by any true trajectory. Indeed, same arguments can prove that there exists $\gamma>0$, such that every $\delta$-pseudo trajectory that comes within $\gamma$ of $\bar{x}_{-n_{3}}$ cannot be $\epsilon$-shadowed by any true trajectory. Without loss of generality, we assume $\delta$ is sufficiently small. Then from Proposition 2.1, almost every $\delta$-pseudo trajectory comes within $\gamma$ of $\bar{x}_{-n_{3}}$. Thus we complete the proof.

Theorem 2.2 is our main result. Note that dimension variability is the key.

\section{EXAMPLES}

Abraham and Smale [1] give an example of an open set of maps on a four dimensional manifold with the following property. There are two disjoint compact invariant sets, $\Lambda_{1}$ (a 2-torus on which the map is the Thom diffeomorphism defined by the linear isomorphism with matrix $\left(\begin{array}{ll}1 & 2 \\ 1 & 1\end{array}\right)$ ), and $\Lambda_{2}$ (a saddle fixed point whose stable manifold is one dimensional), and there are a finite number of trajectories asymptotic to $\Lambda_{1}$ as $n \rightarrow-\infty$ and to $\Lambda_{2}$ as $n \rightarrow \infty$. For a dense set of maps the negative limit sets of these trajectories are each equal to $\Lambda_{1}$ and for another dense set at least one of these trajectories has a negative limit set that is a periodic 
orbit, a proper subset of $\Lambda_{1}$. While they emphasize $\Omega$-stability and do not mention shadowability, it is clear that such trajectories cannot be shadowed by trajectories in nearby systems. It can be shown that the map satisfies our $k$-cone condition with $k=1$ at least for some choices of the parameter. We could adapt Theorem 2.2 as well to this non-attracting case and show the existence of a nonshadowable pseudo trajectory.

In this section we present two other examples. The goal is to show that there exists an open set of maps for which every point is absolutely nonshadowable.

Example 3.1. Kostelich et al. [16] have considered a map on $T^{2}$ given by:

$$
f(y, z)=(2 y \bmod 2 \pi,(y+z)+c \sin (y+z) \bmod 2 \pi)
$$

where $c$ is a parameter in a neighborhood of 0.6 . The Jacobian matrix of the map is:

$$
D f_{(y, z)}=\left(\begin{array}{cc}
2 & 0 \\
1+c \cos (y+z) & 1+c \cos (y+z)
\end{array}\right)
$$

Kostelich et al. [16] have proved that $f$ has a dense trajectory, which implies that $T^{2}$ is an attractor as defined in Section 2; also, $T^{2}$ continues to have a dense trajectory under small $C^{r}$-perturbations, where $r>1$. There are two fixed points of $f:(0,0)$, a source, and $(0, \pi)$, a saddle. These fixed points are hyperbolic and persist under small $C^{r}$-perturbations. Therefore, there exists an open set of maps which have dimension variability.

In contrast, no 2-dimensional diffeomorphism may have dimension variability, because the $\omega$-limit set of a trajectory does not contain a source unless the trajectory is the source itself.

By Theorem 2.2, in order to show nonshadowability it suffices to show that there exists a positively invariant 1 -cone field. The existence follows from the fact that the $y$-coordinate expands by a factor of 2 , more than the expansion of the $z$-coordinate. Specifically, it can be seen as follows. Let $M$ be a constant which is greater than $(1+c) /(1-c)$, A tangent vector $(1, t)$ at $(y, z)$ maps to $(2,(1+t)(1+c \cos (y+z)))$ at $f(y, z)$. If $|t| \leq M$, then $|(1+t)(1+c \cos (y+z))| \leq(1+c)(1+|t|)<2 M$. Therefore $\{(u, v):|v| \leq M|u|\}$ is a positively invariant 1-cone field for $f$. Indeed, it continues to be positively invariant under small $C^{r}$-perturbations. Thus there exists an open set of maps for which every point is absolutely nonshadowable.

We remark that it is also easy to make a three dimensional example in which there is a two dimensional attracting torus which has the described dynamics. This can be done by crossing the above example with a circle on which the map has an attracting fixed point.

Example 3.2. The torus map in Example 3.1 is not one-to-one. However, we can use a technique introduced by Smale [22] to build a diffeomorphism example from an endomorphism one. The idea is to replace the circle map (the $y$-component of Equation (3.1)) by a "solenoid" diffeomorphism on a solid torus $S^{1} \times D^{2}$. Let $M=S^{3} \times S^{1}$, and let $\bar{M}=S^{1} \times D^{2} \times S^{1} . \bar{M}$ is a submanifold (with boundary) of $M$. On $\bar{M}$ we can define coordinates $(t, z, s)$ such that $t \in S^{1}, z \in D^{2}(z$ is a complex number), and $s \in S^{1}$. Define $\bar{g}: \bar{M} \rightarrow \bar{M}$ (see [19], pages 294-298) as $(t, z, s) \mapsto\left(2 t, \frac{1}{4} z+\frac{1}{2} e^{2 \pi t i},(t+s)+c \sin (t+s)\right)$ where $c$ is in a neighborhood of 0.6. $\bar{g}$ can be extended to a global diffeomorphism $g: M \rightarrow M$ such that $\left.g\right|_{\bar{M}}=\bar{g}$. It follows immediately that there is an attractor $A \subset S^{1} \times D^{2} \times S^{1}$, which is 
the Cartesian product of a solenoid attractor in $S^{1} \times D^{2}$ and $S^{1}$. Using similar arguments to those in Kostelich et al. [16], we can prove that $A$ continues to be an attractor. Similarly to Example 3.1, the attractor $A$ has dimension variability and a positively invariant 1-cone field. Therefore Theorem 2.2 implies that every point in the basin of $A$ is absolutely nonshadowable. Indeed, $\bar{g}$ can be extended in such a way that $A$ attracts almost every trajectory in $S^{3} \times S^{1}$. Thus there exists an open set of diffeomorphisms for which every point is absolutely nonshadowable.

We thank Leny Nusse, Lan Wen and Vadim Kaloshin for helpful communications.

\section{REFERENCES}

[1] Ralph H. Abraham and Stephen Smale, Nongenericity of $\Omega$-stability, Global analysis (Proceedings of Symposia in Pure Mathematics, vol. 14, 1968), vol. 14, American Mathematical Society, 1970, pp. 5-8. MR 42:6867

[2] D. V. Anosov, Geodesic flows on closed riemann manifolds with negative curvature, Proceedings of the Steklov Institute of Mathematics 90 (1967). MR 39:3527

[3] Michael Benedicks and Lennart Carleson, The dynamics of the Hénon map, Annals of Mathematics(2) 133 (1991), no. 1, 73-169. MR 92d:58116

[4] Rufus Bowen, w-limit sets for Axiom A diffeomorphisms, Journal of Differential Equations 18 (1975), no. 2, 333-339. MR 54:1300

[5] Shui-Nee Chow and Kenneth J. Palmer, On the numerical computation of orbits of dynamical systems: the one-dimensional case, Journal of Dynamics and Differential Equations 3 (1991), no. 3, 361-379. MR 92h:58057

[6] _ On the numerical computation of orbits of dynamical systems: the higherdimensional case, Journal of Complexity 8 (1992), no. 4, 398-423. MR 93k:65118

[7] Brian A. Coomes, Hüseyin Koçak, and Kenneth J. Palmer, Periodic shadowing, Chaotic Numerics, Contemporary Mathematics Series 172., American Mathematical Society, Providence, Rhode Island, 1994, pp. 115-130. MR 95i:58128

[8] _ Shadowing in discete dynamical systems, Six Lectures on Dynamical Systems, World Scientific Publishing, River Edge, New Jersey, 1996, pp. 163-211. MR 98d:58133

[9] Ethan M. Coven, Ittai Kan, and James A. Yorke, Pseudo-orbit shadowing in the family of tent maps, Transactions of the American Mathematical Society 308 (1988), no. 1, 227-241. MR 90b:58236

[10] William Feller, An introduction to probability theory and its applications, John Wiley \& Sons, Inc., 1957. MR 19:466a

[11] Jacques Hadamard, Sur l'itération et les solutions asymptotiques des équations différentielles, Bulletin de la Société Mathématique de France 29 (1901), 224-228.

[12] Stephen M. Hammel, James A. Yorke, and Celso Grebogi, Do numerical orbits of chaotic dynamical processes represent true orbits?, Journal of Complexity 3 (1987), no. 2, 136-145. MR 88m:58115

[13] _ Numerical orbits of chaotic processes represent true orbits, Bulletin of American Mathematical Society 19 (1988), no. 2, 465-469. MR 89m:58180

[14] Anatole Katok and Boris Hasselblatt, Introduction to modern theory of dynamical systems, Cambridge University Press, 1995. MR 96c:58055

[15] Yuri Kifer, Random perturbations of dynamical systems, Birkhauser, 1988. MR 91e:58159

[16] Eric J. Kostelich, Ittai Kan, Celso Grebogi, Edward Ott, and James A. Yorke, Unstable dimension variability: A source of nonhyperbolicity in chaotic systems, Physics and dynamics between chaos, order, and noise (Berlin, 1996) Phys. D 109 (1997), no. 1-2. 81-90. MR 98a: 58110

[17] P. J. Myrberg, Sur l'itération des polynomes réels quadratiques, Journal de Mathématiques Pures et Appliquées (9) 41 (1962), 339-351. MR 28:5171

[18] Leon Poon, Celso Grebogi, Tim Sauer, and James A. Yorke, Limits to deterministic modeling, Preprint.

[19] Clark Robinson, Dynamical systems : stability, symbolic dynamics, and chaos, CRC Press, Inc., 1995. MR 97e:58064

[20] Tim Sauer, Celso Grebogi, and James A. Yorke, How long do numerical chaotic solutions remain valid?, Physical Review Letters 79 (1997), 59. 
[21] Tim Sauer and James. A. Yorke, Rigorous verification of trajectories for the computer simulation of dynamical systems, Nonlinearity 4 (1991), no. 3, 961-979. MR 93a:58104

[22] Stephen Smale, Differentiable dynamical systems, Bulletin of the American Mathematical Society 73 (1967), 747-817. MR 37:3598

[23] Stephen Smale and Robert F. Williams, The qualitative analysis of a difference equation of population growth, Journal of Mathematical Biology 3 (1976), no. 1, 1-4. MR 54:2251

[24] Lai-Sang Young, Stochastic stability of hyperbolic attractors, Ergodic Theory and Dynamical Systems 6 (1986), 311-319. MR 88a:58160

Institute for Physical Science and Technology, and Department of Mathematics, University of Maryland, College Park, Maryland 20742

E-mail address: gcyuan@ipst.umd.edu

E-mail address: yorke@ipst.umd.edu 